\title{
LA ADAPTACIÓN AL ESPACIO EUROPEO DE EDUCACIÓN SUPERIOR COMO ESCENARIO PARA LA REFLEXIÓN SOBRE EL PROCESO DE ENSEÑANZA-APRENDIZAJE EN EL ÁMBITO UNIVERSITARIO: ALGUNAS PROPUESTAS PARA UN CAMBIO SIGNIFICATIVO
}

\author{
THE EUROPEAN HIGHER EDUCATION AREA (BOLONIA PROCESS) AS A \\ CONTEXT FOR REFLECTION ABOUT TEACHING-LEARNING PROCESSES AT \\ UNIVERSITY LEVEL: SOME PROPOSALS FOR A SIGNIFICANT CHANGE
}

\author{
$M^{a}$ Ángeles Caballero Hernández-Pizarro* \\ Universidad Complutense de Madrid
}

\section{RESUMEN}

Hemos superado el ecuador temporal de un proceso de convergencia sobre el que podemos escuchar argumentos a favor y en contra, pero que indiscutiblemente esta configurando un nuevo escenario para la docencia universitaria en Europa. Con este artículo se pretende invitar a profesores y orientadores a adoptar una actitud crítica y reflexiva ante los cambios que se están produciendo, y que implican la asunción de nuevos papeles en los que docentes y orientadores deberán trabajar juntos. Sólo desde esta actitud crítica y colaborativa se logrará un verdadero cambio significativo, que permitirá alcanzar de forma eficaz las metas del proceso de Bolonia.

Palabras clave: docencia y orientación universitaria, estrategias de enseñanza, aprendizaje significativo, Espacio Europeo de Educación Superior.

\footnotetext{
* Dra. Ma Ángeles Caballero Hernández-Pizarro. Profesora titular del Departamento de Métodos de Investigación y Diagnóstico en Educación, de la Facultad de Educación de la UCM. Líneas de investigación: Estrategias de enseñanza-aprendizaje: identificación de estrategias, dificultades lecto-escritoras (ayuda a alumnos inmigrantes en procesos de castellanización). Orientación profesional: elaboración de perfiles de intereses profesionales, diseño de herramientas para facilitar la toma de decisiones. Identificación y mejora de competencias básicas (maestros, psicopedagogos). E-mail: mariachp@edu.ucm.es
} 


\begin{abstract}
In the project of creating a European Higher Education Area, we have run out part of the time scheduled to achieve this objective. This process — letting aside arguments in favour or against - is undoubtedly creating a new background (scenery) for European Universities. The aim of this article is to promote, among teachers and counsellors, a reflective and critical attitude towards the changes this new context implies. Both professionals will have to assume new roles, in which cooperation will be necesary. Only then, a critical and collaborative attitude will be possible to achieve the goals of the Bolonia process.
\end{abstract}

Key words: teaching-learning processes at the University, counselling in higher education, teaching strategies, significant learning, Bologna Process.

\title{
Algunas pinceladas sobre el Espacio Europeo de Educación Superior
}

En 1999, un grupo de Ministros de Educación de 29 países de la Unión Europea, reunidos en la ciudad de Bolonia, se comprometen en un interesante y complejo proyecto: crear un Espacio Europeo de Educación Superior. Las metas compartidas que justifican y dirigen este proceso quedaron recogidas en el informe del Consejo de la Unión Europea (Council of the European Union) ${ }^{1}$, firmado el 14 de febrero de 2001 en la ciudad de Bruselas. En el citado informe se destacan "ambiciosas" propuestas: mejorar la calidad y efectividad de la educación, facilitar el acceso a la educación y a los diferentes sistemas educativos, adaptar la educación y la formación a un mundo en constante cambio en el que el proceso de enseñanza-aprendizaje se extiende durante todo el proceso vital (world of lifelong learning), fortalecer la cooperación entre los países europeos, o facilitar la adquisición de competencias clave (key competentes).

Establecer las metas es sólo el principio de un proceso de cambio cuya fecha límite ha sido fijada, en nuestro país, para el 2010. Se ha superado, por tanto, el ecuador de este recorrido, y se han tomado importantes decisiones que afectan a la estructura y organización de las enseñanzas universitarias que pueden sintetizarse en tres:

- La organización de los estudios universitarios en dos ciclos: el grado (R.D. 55/2005, de 21 de enero) y el postgrado (R.D. 56/2005, de 21 de enero).

- El establecimiento de un suplemento europeo al título, con el que se pretende lograr una mayor transparencia y facilitar los procesos de reconocimiento de estudios (R.D. 1044/2003, de 1 de agosto).

- La adopción de un sistema compartido, los ECTS, que nos permita contabilizar el trabajo que los estudiantes necesitan realizar para superar sus estudios universitarios. (Tal vez sea más correcto, como se argumentará más adelante, hablar de contabilizar el proceso de enseñanza-aprendizaje). Con este sistema se pretende no sólo consensuar una unidad de medida que facilite los procesos de intercambio universitario - equivalencia de estudios - , sino reconocer la complejidad de los procesos de enseñanza-aprendizaje (R.D. 1125/2003, de 5 de septiembre).

1. Se puede consultar este informe en la siguiente dirección electrónica: http:/europa.eu.int/comm/education/ policies/2010/doc/rep_fut_obj_en.pdf 
Los cambios encaminados a la construcción del EEES constituyen, en definitiva, un nuevo "escenario" universitario que invita a la reflexión sobre la universidad que queremos (Vieira y Vidal, 2006: 75). Esta reflexión ha generado argumentos a favor y en contra, tanto desde el colectivo de profesores como de alumnos ${ }^{2}$. Desde las universidades se ha trabajado para que el ambos colectivos estén debidamente informados — repárese, a modo de ejemplo, en las páginas "web" de las universidades en las que aparece siempre un enlace relacionado con este tema-, y también se han venido desarrollando grupos pilotos que faciliten esta transición. No es nuestra intención hacer una relación exhaustiva de todas estas iniciativas, pero sí considerar la posibilidad de que todos estos cambios se estén produciendo de una manera mecánica, sin suponer un verdadero cambio significativo para sus principales protagonistas, en concreto, para los profesores universitarios.

\section{La adaptación al EEES: ¿por qué un cambio significativo?}

La regulación legislativa de los cambios descritos no garantiza que se vaya a producir una transformación o adaptación en el modo de entender y desarrollar la función docente universitaria. El cambio puede abordarse desde dos posicionamientos estratégicos:

- Un planteamiento superficial, que busca soluciones a corto plazo, y que por tanto no va a afectar a nuestro modo de vida.

- Un planteamiento reflexivo y crítico, que busca soluciones duraderas, y que necesariamente exige la reorganización y transformación de nuestros planteamientos.

Es esta segunda interpretación, este cambio significativo como lo denominarían los psicólogos cognitivistas (Norman, Rumelhart, Ausubel, etc.), exige adaptación (tal y como recoge la ecuación de Piaget: Asimilación + acomodación = adaptación), el que todos los agentes implicados en la educación universitaria — alumnos, docentes y orientadores- deben procurar y conseguir.

¿Qué tipo de cambio — superficial o significativo-, estamos experimentando los profesores universitarios? Para responder a esta pregunta, tomemos como ejemplo la implantación del nuevo sistema europeo de créditos o ECTS. Estas siglas esconden la expresión European Credit Transfer System (sistema europeo de transferencia de créditos), sistema diseñado para valorar el volumen de trabajo efectivo del estudiante, de modo que sea posible comparar el trabajo académico de los estudiantes en los programas de intercambio (p.e. el programa Erasmus). El sistema establece que un crédito suponer entre 25 y 30 horas de trabajo, y que la carga de trabajo para un curso académico es de 60 créditos. Para algunos profesores, la incorporación de los ECTS supone sólo una medida administrativa que obliga a realizar algunos cálculos. Dado que el actual plan de estudios se contabiliza en horas de docencia del profesor, será necesario realizar una reconversión numérica para poder responder a la pregunta ¿a cuántos ECTS equivale mi actual "crédito"?). Para estos profesores sólo se estaría produciendo un cambio superficial, porque no se están preguntando por las razones que justifican y hacen necesario este cambio (asimilación), ni están transformando

2. Recientemente, 11 y 12 de mayo, se ha celebrado en Santander un "Encuentro Interuniversitario de estudiantes para el estudio y la implantación del EEES. 
su sistema de enseñanza como consecuencia de la asimilación de estos cambios (acomodación). Esta actitud es insuficiente como se justificará a continuación.

El verdadero cambio, el cambio significativo, nos tiene que llevar a preguntarnos por qué ahora hablamos de "horas de trabajo del alumno" y no de "horas de clase", y descubriremos que tras los ECTS se esconde una importante revolución metodológica que plantea una nueva atribución de significados de las tareas docentes y discentes, y que tiene como consecuencia una nueva contabilidad del sistema de enseñanza-aprendizaje, en la que se otorga valor a elementos ignorados en nuestro habitual sistema de "contabilidad docente". El crédito ECTS supone básicamente trasladar el foco de atención del profesor al alumno. El sistema actual de "medida de la docencia" contabiliza el número de horas de clase que imparte el profesor; el sistema ECTS lo que pretende es estimar la carga de trabajo del estudiante necesaria para la consecución de los objetivos del programa. Así queda recogido en el correspondiente Real Decreto:

"En esta unidad de medida se integran las enseñanzas teóricas y prácticas, así como otras actividades académicas dirigidas, con inclusión de las horas de estudio y de trabajo que el estudiante debe realizar para alcanzar los objetivos educativos." (RD 1125/2003, de 5 de septiembre, art. 3).

Tras las adaptaciones legislativas hemos de descubrir las verdaderas consecuencias para la docencia universitaria de este nuevo escenario docente que podríamos sintetizar en:

- Plantear el proceso de enseñaza como un sistema que integra los procesos de aprendizaje — "sistema de enseñanza-aprendizaje"—, es decir, dejar de considerar nuestra función docente como una tarea unidireccional (enseñar), para planteárnosla como una tarea bidireccional (enseñanza-aprendizaje), que nos lleva a reconocer el protagonismo del alumno en este proceso.

- Revisar nuestro papel como docentes, renunciando tal vez a nuestro habitual papel de "dictadores" de apuntes, para transformarnos en facilitadores del aprendizaje, incorporando nuevos sistemas de enseñanza e integrando la función tutorial.

\section{Replantearse el sistema docente}

Los planteamientos que justifican el nuevo sistema de valoración de la docencia no hacen sino reflejar las exigencias y demandas de la sociedad actual. Tal y como se señala en el informe Universidad 2000:

"Los cambios y las nuevas demandas sociales hacen que en la universidad el aprendizaje adquiera progresivamente más protagonismo: en el binomio enseñanza-aprendizaje es imprescindible poner el énfasis en este último aspecto, entendiendo la enseñanza como un sistema para facilitarlo; como un medio más que como un fin en sí mismo." (Informe Universidad 2000: 16).

Se insiste en la necesidad de otorgar al alumno un mayor protagonismo en el desarrollo del proceso de enseñanza aprendizaje, pero no se trata de "dar la vuelta a la tortilla", sino de lograr un cambio de actitud en el profesor universitario, que no puede limitar su preocupación al contenido científico, sino que ha de reflexionar sobre el modo como éste es aprendido y el modo en que puede ser enseñado. Este es el planteamiento implícito en los ECTS 
cuando se afirma que es necesario valorar el "volumen de trabajo" del estudiante. Para lograr este propósito, necesito especificar en qué consiste ese trabajo, y éste, aunque parezca una simplicidad, no es otro que el de "estudiar para aprender". Pretender valorar el trabajo del estudiante supone "situarse en la perspectiva del que aprende y tener en cuenta sus estados mentales" (Delval, 2000: 26), supone admitir que los profesores deben hacer conjeturas sobre los procesos mentales de sus alumnos, estimar su estado de conocimiento, determinar las dificultades que pueden encontrarse, y muy especialmente, preguntase sobre cómo pueden intervenir de forma eficaz para ayudar a sus alumnos a aprender.

¿Qué tipo de enseñanza es más adecuado para lograr que el alumno sea el protagonista de su propio aprendizaje?

Desde la institución universitaria, y a la luz de la reforma iniciada en el proceso de Bolonia, queda claro que lo que se pretende es dotar a los alumnos de unos conocimientos, destrezas y valores que les permitan una adecuada integración en la vida profesional. El propósito de la formación universitaria será explicitado en términos de competencias. La identificación de estas competencias ¿tiene alguna implicación metodológica? o más específicamente, ¿orienta la formación universitaria hacia un determinado modo de aprender? Para poder responder a esta pregunta, hemos de reflexionar sobre el modo como se produce el aprendizaje y las tipologías de aprendizaje derivadas. Distintos estudiosos del tema (Ausubel, Novak, Gowin, Piaget, Vygotsty ... por citar algunos de los autores más relevantes), han profundizado en el estudio de los distintos modos de aprender. Centremos esta reflexión clarificadora sobre los tipos de aprendizaje en torno a dos grandes ejes:

- Eje significativo - mecánico (grado de significación y elaboración de contenidos).

El aprendizaje significativo, concepto central de la teoría de Ausubel, se define como "un proceso a través del cual una misma información se relaciona, de manera no arbitraria y sustantiva (no literal), con un aspecto relevante de la estructura cognitiva del individuo" (Moreira, 2000: 11). Es decir, la nueva información ha de interactuar con la que ya se posee, de modo que se genere una nueva información que realmente tenga significado para el individuo. En términos muy parecidos se expresaba Piaget (1971) cuando definía el aprendizaje como el resultado de un proceso de asimilación y acomodación (modificación de la estructura congitiva), que da como resultado nuevos esquemas. Por oposición a este aprendizaje orientado a la construcción de nuevos significados, Ausubel define el aprendizaje mecánico (o automático) "como aquel en que las nuevas informaciones se aprenden prácticamente sin interacción con conceptos relevantes existentes en la estructura cognitiva" (Moreira, 2000: 12), sin ligarse o interactuar con ninguna estructura cognitiva preexistente, por lo que la nueva información es almacenada de manera arbitraria y literal, sin apenas elaboración.

\section{- Eje descubrimiento - recepción}

Completa la caracterización del aprendizaje desde la perspectiva del modo en que la información es presentada al alumno, o el modo como el alumno accede a la información. Según Ausubel "en el aprendizaje receptivo lo que debe aprenderse se le presenta al aprendiz en su forma final, mientras que en el centrado en el descubrimiento, el contenido principal objeto de aprendizaje debe ser descubierto por él mismo." (Moreira, 2000: 13).

Conocidas las opciones, nuestra elección deberá tener siempre presente que "en definiti$v a$, es el aprendiz el que tiene que tomar sus nuevos conocimientos y que el tutor (profesor) 
no puede transmitírselos hechos, sino solamente ayudarle a construirlos" (Delval, 2000: 29). Tal vez sea preciso recurrir a la Teoría Educativa de Novak (1981), para encontrar argumentos que nos ayuden a tomar una decisión sobre el tipo de aprendizaje que consideramos más adecuado facilitar en nuestros alumnos (y en nosotros mismos). La premisa básica de la teoría de Novack es que los seres humanos hacen tres cosas: piensan, sienten y actúan. El aprendizaje significativo es sólo una parte integrante de este proceso en el que se combinan un conjunto de experiencias (cognitivas, afectivas y psicomotoras), que contribuyen al engrandecimiento (empowerment) del individuo para enfrentarse a la vida diaria. "Cualquier evento educativo implica una acción para intercambiar significados y sentimientos entre el profesor y el alumno" (Moreira, 2000: 40), lo que implica que los significados del aprendizaje son contextuales.

La consideración del aprendizaje no sólo como generador de un desarrollo cognitivo, sino como vía hacia el enriquecimiento personal en al menos tres dimensiones: cognitiva, afectiva y social, nos lleva a incluir nuevos requisitos en el aprendizaje que queremos facilitar en nuestros alumnos, requisitos que podríamos sintetizar en dos: crítico y reflexivo. Si desde la formación universitaria pretendemos formar profesionales competentes, entendiendo esa competencia como "la aptitud para enfrentar eficazmente una familia de situaciones análogas, movilizando a conciencia y de manera a la vez rápida, pertinente y creativa, múltiples recursos, valores, aptitudes, esquemas de percepción, de evaluación y de razonamiento" (Perrenoud, 2001:509), es necesario que definamos un sistema de enseñanzaaprendizaje en el que el profesor actúe como mediador o facilitador de un aprendizaje significativo y crítico que permita un desarrollo integral del alumno (cognitivo, afectivo y social), que le capacite para el desempeño competente de su profesión. Pero no nos gustaría que la formación se agotara en los planteamientos productivos, y que considerase que esa integración profesional forma parte del proceso de integración y enriquecimiento personal y social de nuestros alumnos.

\section{Estrategias metodológicas que potencian el aprendizaje}

Las reflexiones y argumentos teóricos expuestos hasta el momento nos han proporcionado materiales muy útiles para poder construir la respuesta a una pregunta importante: ¿cómo potenciar el aprendizaje de nuestros alumnos? o dicho en términos más precisos ¿qué debe hacer el profesor para que el alumno aprenda significativamente? En definitiva, lo que necesitamos es diseñar una buena metodología didáctica. Precisar lo que es la metodología más allá de estos términos resulta bastante complejo. Es necesario indicar que bajo esta denominación se esconde un sistema de decisiones sobre distintas cuestiones que afectan y configuran nuestra tarea docente. Nuestra postura coincide básicamente con la expresada por Zabalza (2003: 103):

"El término método constituye un constructo quizás excesivamente amplio e internamente heterogéneo. Se ha utilizado como una especie de cajón de sastre en el que caben muchos componentes: la forma de abordar los contenidos, los estilos de organización del grupo de alumnos, el tipo de tareas o actividades, el estilo de relación entre las personas, etc."

Lo más correcto es abordar la solución del problema en dos niveles de concreción: 


\section{- $1^{\text {er }}$ nivel (general):}

Delimitar o configurar nuestro "sistema" de enseñanza, el conjunto de "orientaciones metodológicas o grandes líneas matrices" (Zabalza, 2003: 189), desde las que nos planteamos nuestra intervención docente. Hemos optado por la denominación "estilo de enseñanza". Tomando como base la propuesta del citado autor, consideramos que nuestra manera de enfrentar la tarea docente (estilo docente), es la combinación de nuestras respuestas (opción o decisión tomada), a las siguientes preguntas (referidas a distintos componentes de la docencia). Representamos nuestra propuesta en la tabla 1.

TABLA 1: Sistema de Enseñanza. Elaboración propia.

\begin{tabular}{|c|c|}
\hline Aspectos de la docencia & Opciones (posibles respuestas) \\
\hline ¿Cómo accede (el alumno) al conocimiento? & $\begin{array}{l}\text { - Exposición (lección magistral). } \\
\text { - Descubrimiento. }\end{array}$ \\
\hline $\begin{array}{l}\text { ¿Qué tipo de comunicación se establece entre profesor y } \\
\text { alumno? }\end{array}$ & $\begin{array}{l}\text { - Directa o presencial. } \\
\text { - Semi-presencial. } \\
\text { - No presencial. }\end{array}$ \\
\hline $\begin{array}{l}\text { ¿Qué tipo de control realizo sobre el proceso de } \\
\text { aprendizaje? }\end{array}$ & $\begin{array}{l}\text { - Directivo. } \\
\text { - Guiado. } \\
\text { - Autónomo. }\end{array}$ \\
\hline $\begin{array}{l}\text { ¿Cómo es la relación } \\
\text { (Interacción) profesor-alumno? }\end{array}$ & $\begin{array}{l}\text { - Dogmática (dependencia). } \\
\text { - Colaborativa (independencia). }\end{array}$ \\
\hline
\end{tabular}

Un profesor, por ejemplo, puede decantarse por un sistema de acceso a la información basado en el descubrimiento, con un control moderado sobre el estudiante (guiado), que exige una comunicación semi-presencial y una relación colaborativa entre profesor y alumno. También supondría combinar el trabajo individual con el trabajo en grupo. Es posible buscar una denominación específica para cada uno de estos sistemas de acción; de hecho, existen propuestas interesantes. A modo de ejemplo, podemos citar la propuesta de Tejada (2001), que diferencia tres grandes categorías: método magistral, trabajo autónomo y trabajo por grupos.

¿Cuál sería el estilo docente más adecuado para potenciar el aprendizaje autónomo?

Si respondemos que "todos", estaríamos dando una respuesta parcial. En realidad todos pueden ser válidos dependiendo de nuestras intenciones, del momento del proceso de aprendizaje en que nos encontremos y del periodo evolutivo en que se hallen nuestros alumnos. Podemos concluir diciendo que, debemos adoptar un estilo de enseñanza flexible y crítico, es decir, no "empeñarnos" en desarrollar siempre el mismo y único estilo, sino realizar todos los ajustes y cambios necesarios para lograr los objetivos propuestos y para ajustarnos a las exigencias del contexto. 


\section{- $2^{\circ}$ nivel: (específico):}

Nuestro estilo de enseñanza se materializará en una serie de acciones, preferiblemente justificadas y organizadas; a estas acciones específicas podemos denominarlas estrategias o técnicas. Ambas palabras hacen referencia a procedimientos de acción, la diferencia entre ambas esté en el control. Como señala Pozo (1999), las técnicas "son rutinas de acción automatizadas", mientras que las estrategias exigen Aun uso deliberado y planificado. Entre ambos términos se establece una diferencia de grado: "Técnicas y estrategias son formas progresivamente más complejas de utilizar un mismo procedimiento" (Pozo, 1999: 300). La diferencia no está, por tanto, en qué se hace, sino en cómo se hace, o mejor dicho, cómo se usa, pudiéndose utilizar un procedimiento (conjunto de acciones) de modo técnico, es decir, rutinario, sin planificación ni control, o de un modo estratégico (Monereo et al., 1994). Podemos hablar, por tanto, de estrategias didácticas que se concretan en técnicas (e incluso en instrumentos), o simplemente hablar de técnicas didácticas y considerar que se puede hacer un uso técnico o estratégico de las mismas. En un nivel puramente estratégico, podemos considerar que, como señala Mayer (2002), para promover el aprendizaje significativo es necesario reunir tres condiciones: seleccionar adecuadamente la información, organizar el conocimiento e integrar los conocimientos aprendidos. Desde esta base, habremos de considerar que, como profesores, deberemos elaborar estrategias que ayuden al estudiante a:

- localizar y escoger la información adecuada.

- ordenar (clasificar y jerarquizar) el conocimiento.

- relacionar los conocimientos nuevos con los conocimientos previos, de manera que se produzca reestructuración (elaboración) del conocimiento.

¿Cuáles son las técnicas que van a permitirnos proporcionarle esta ayuda, y de las que intentaremos hacer un "uso estratégico"?

No es posible presentar ninguna técnica o estrategia como la más adecuada; nuestro trabajo consiste nuevamente en justificar la elección y uso de una u otra estrategia. Disponer de una relación ordenada y estructurada de las distintas propuestas puede facilitar el proceso de toma de decisiones. Esta propuesta debe organizarse con referencia a elementos estructuradores que a los profesores nos faciliten la toma de decisiones. Recogiendo los argumentos teóricos que se han venido exponiendo, se propone una estructura de tabla con una doble entrada: por un lado, tomaremos como referencia las tres grandes estrategias que, según Mayer, habremos de trabajar con nuestros alumnos para lograr el aprendizaje significativo (progresión vertical, y en la progresión vertical, diferenciaremos entre técnicas centradas en la enseñanza (el protagonista es el profesor), y técnicas centradas en el aprendizaje (el protagonista es el alumno). La propuesta final queda recogida en la tabla 2.

\section{Consideraciones finales: la necesidad de una estrecha colaboración entre orientadores y docentes}

En la naturaleza funciona el principio de la inercia o tendencia a mantenerse en el mismo estado hasta que se produce una circunstancia (fuerza) que nos obliga a modificar dicho estado (cambiar). ¿Son los cambios propuestos por el EEES suficientemente "fuertes" para 
TABLA 2: Estrategias, técnicas e instrumentos que podemos utilizar para potenciar el aprendizaje significativo. Elaboración propia.

\begin{tabular}{|l|l|l|}
\hline Estrategias & \multicolumn{1}{|c|}{$\begin{array}{c}\text { Técnicas centradas en la } \\
\text { enseñanza (profesor) }\end{array}$} & $\begin{array}{l}\text { Técnicas centradas en el } \\
\text { aprendizaje (alumno) }\end{array}$ \\
\hline \multirow{5}{*}{ Selección } & & $\begin{array}{l}\text { Toma de apuntes. } \\
\text { Lectura de textos. } \\
\text { Búsqueda de información: } \\
\text { * buscadores (internet) } \\
{ }^{*} \text { catálogos }\end{array}$ \\
\hline \multirow{5}{*}{ Organización } & $\begin{array}{l}\text { Elaboración de: } \\
\text { - Esquemas. }\end{array}$ \\
& $\begin{array}{l}\text { Nota: es de gran ayuda para el } \\
\text { profesor utilizar técnicas de } \\
\text { organización de la información para } \\
\text { la elaboración de sus exposiciones. }\end{array}$ & $\begin{array}{l}\text { - Resúmenes. } \\
\text { - Árbol o mapa semántico. } \\
\text { - Redes conceptuales. } \\
\text { - Mapas conceptuales. } \\
\text { - Mapas mentales. } \\
\text { - V de Gowin. }\end{array}$ \\
\hline
\end{tabular}

obligarnos a cambiar el rumbo de nuestras acciones? La necesidad de abandonar nuestros modos habituales de comportamiento aprovechando la oportunidad de análisis y reflexión que nos brinda el "espacio europeo" debe ser aceptada tanto por alumnos como por profesores. Los profesores, debemos abandonar nuestro punto de vista y pensar desde la perspectiva del alumno, rediseñando nuestras estrategias de enseñanza, pero los alumnos también deben abandonar sus sistemas tradicionales de aprendizaje, basados en la memorización y acostumbrados al directivismo, lo que les encasilla en una actitud receptiva y pasiva.

Profesores y alumnos han de enfrentarse a nuevos compromisos personales y profesionales. El profesorado, acostumbrado a valorar la calidad de su docencia en relación exclusiva a la claridad y estructura de sus exposiciones magistrales, y a concebir su relación personal con el alumno desde planteamientos directivos y escasamente individualizados, deberá incorporar a sus preocupaciones docentes la planificación de tareas de aprendizaje dirigido y la atención personal en las tutorías con un carácter más sistemático y frecuente. El diseño y desarrollo de planes y programas deberá superar los planteamientos individuales para ser responsabilidad de los departamentos y grupos de profesores, obligando a los docentes a abandonar sus dominios individuales. El alumno deberá comprometerse en un aprendizaje más responsable y autónomo, a gestionar su tiempo y a trabajar en equipo.

En todo este proceso, el papel de la orientación universitaria es esencial. Profesores y alumnos necesitan ser apoyados para que, desde una actitud crítica y colaborativa, puedan afrontar con éxito los retos de este nuevo contexto, y asumirlos de forma significativa. Los equipos de orientadores en las distintas instituciones universitarias europeas ya están poniendo en marcha programas en los que se incluyen talleres y seminarios orientados tanto a alumnos como a profesores, con resultados muy positivos ${ }^{3}$.

3. A modo de ejemplo, remitimos a las experiencias desarrolladas por la Universidad de Tampere (Finlandia) y de Hamburgo (Alemania), que fueron presentadas en la conferencias de Fedora-Psyche (2007). 
Debemos considerar también, que cualquier iniciativa de apoyo que se plantee desde los servicios de orientación universitarios puede resultar inútil si los docentes no comparten los mismos principios de acción. De este modo, un interesante taller sobre "Estrategias de aprendizaje" no tendrá apenas valor si el profesor se limita a exigir a sus alumnos que memoricen los contenidos por él presentados. Es por ello que insistimos en la necesidad de que orientadores y docentes trabajen de forma colaborativa, pues en definitiva comparte un mismo propósito: ayudar a los estudiantes en su desarrollo personal y profesional.

Profesores, alumnos y orientadores han de reconstruir la docencia universitaria para crear una verdadera comunidad de aprendizaje. Se trata, en definitiva, de un proceso de democratización de la docencia, reconociendo para todos los elementos personales el mismo grado de compromiso y responsabilidad: el profesor y el orientador asumirán el papel de "facilitadores". Gaarder, en su obra "El mundo de Sofía", lanzaba la siguiente acusación:

"La gran diferencia entre un maestro (de escuela) y un auténtico filósofo es que el maestro cree que sabe un montón e intenta obligar a sus alumnos a aprender. Un filósofo intenta averiguar las cosas junto con los alumnos" (Gaarder, 1994: 84).

El colofón de esta argumentación nos lleva a aceptar el reto de Gaarder. Invitamos a orientadores y docentes universitarios a sentirse filósofos, y poder responder que los orientadores y profesores universitarios no se diferencian de los filósofos.

\section{Referencias bibliográficas}

Delval, J. (2000). Aprender en la vida y en la escuela. Madrid: Morata.

Eerora, S. y Nieminen, J. (2007). "Study conunselling psychologist - A developer of Higher Education". Comunicación presentada a Fedora-Psyche Conference 2007, Creta, Grecia.

Figge, P. (2007). "Developing personal study competence. Effects of Soft-Skill Seminars for Students". Comunicación presentada a Fedora-Psyche Conference 2007, Creta, Grecia.

Gaarder, J. (1994). El mundo de Sofia. Madrid: Siruela.

Mayer, R. E. (2002). Psicología de la educación. El aprendizaje de las áreas de conocimiento. Madrid: Prentice-Hall.

Monereo et al. (1994). Estrategias de enseñanza y aprendizaje. Formación del profesorado y aplicación en el aula. Barcelona: Graó.

Moreira, M. A. (2000). Aprendizaje s ignificativo: teoría y práctica. Madrid: Visor.

Novak, J. D. (1981). Teoría y práctica de la educación. Madrid: Alianza.

Parcerisa, A. (2004). Nova estructura del Pla Docent a la Universidat de Barcelona. Barcelona: Universidad de Barcelona.

Perrenoud, P. (2001). La formación de los docentes en el siglo XXI. Revista de Tecnología Educativa, XIV (3), 503-523.

Piaget (1970). Psicología y epistemología. Barcelona: Ariel.

Pujol y Fons (1981). Los métodos en la enseñanza universitaria. Pamplona: Eunsa.

Pozo, J. I. (1999). Aprendices y maestros. La nueva cultura del aprendizaje. Madrid: Alianza Editorial.

Rodríguez Moreno, M. L. (2000). Hacia una nueva orientación universitaria. Barcelona: Edicions Universitat de Barcelona. 
RD 1125/2003, de 5 de septiembre, por el que se establece el sistema europeo de créditos.

Santos Guerra (1990): Mañana examen. Barcelona. Graó.

Tejada, J. (2001): Planificación de acciones de formación ocupacional: estrategias metodológicas. (Documento mimeografiado). Departamento de Pedagogía i Didáctica: Universidad Autónoma de Barcelona.

V.V.A.A. (2000). Informe Universidad 2000. Recuperado el 2 de septiembre de 2007 en http://www. crue.org/informeuniv2000.htm.

V.V.A.A. (2004). Documento de apoyo para la adaptación de experiencias piloto al Espacio Europeo de Educación Superior. Madrid: Universidad Complutense de Madrid.

Vieira, M. J. y Vidal, J. (2006). "Tendencias de la Educación Superior Europea e implicaciones para la orientación universitaria". Revista Española de Orientación y Psicopedagogía (REOP), 17, 1, 75-97.

Zabalza, M. A. (2003). Competencias docentes del profesorado universitario: calidad y desarrollo profesional. Madrid: Narcea.

Fecha de recepción: 12-11-05

Fecha de revisión: 11-02-07

Fecha de aceptación: 07-11-07 